\section{Organschaft}

Die Zeitschrift ist amtliches Organ der Bundesanstalt für Materialforschung und -prüfung sowie Organ des Normenausschusses Materialprüfung im DIN

\section{Herausgeber}

BAM Bundesanstalt für Materialforschung und -prüfung

DGZfP Deutsche Gesellschaft für Zerstörungsfreie Prüfung

DVM Deutscher Verband für Materialforschung und -prüfung

VDI-Gesellschaft Werkstofftechnik

\section{Aufsatz-Redaktion Berlin}

Dr.-Ing. Dietmar Aurich, Ltd. Dir. u.

Prof. (verantwortlich)

Dr. Manfred P. Hentschel

Dr. Jürgen Sickfeld

Dr. Uta Völkel

BAM, Unter den Eichen 87

D-1000 Berlin 45

Telefon: 0 30/8104-1

Telefax: 0 30/8112029

\section{Redaktion Darmstadt}

Dr.-Ing. Thomas Stöckermann

(verantwortlich)

Dipl.-Ing. Claudia Treffert

Carl Hanser Verlag

Marburger Straße 13

D-6100 Darmstadt

Telefon: 0 6151/70 0950

Telefax: $06151 / 700948$

Beiträge und Mitteilungen für den redak tionellen Inhalt sind an die Redaktion Darmstadt zu senden. Fachaufsätze (Originalbeiträge) an die Aufsatzredaktion Berlin.

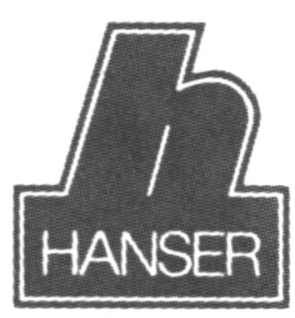

Carl Hanser Verlag

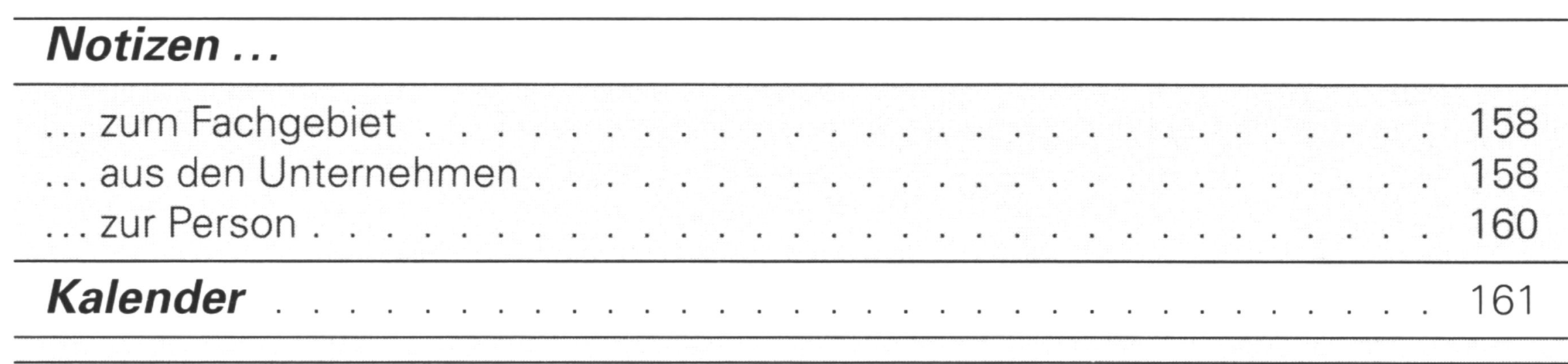

Aufsätze

M. Tietze: Computertechnik und Bildverarbeitung bei der

Wirbelstromprüfung an Flugzeugen .

M. Stadthaus, H.-M. Thomas, H. Haeger: Anforderungen an

Bildverarbeitungsanlagen zur automatischen Auswertung bei der

Magnetpulver- und Eindringprüfung . . . . . . . . . . . . . . . . . 166

M. Platte, A. Ries: Ultraschall-Prüfköpfe für extreme Temperaturen . . . . 170

R. Rosenberg: Automatische und umfassende Korrosionsprüfung an

Rohrleitungen und Tanks

A. B. Doyum: Acousto-Ultrasonic Technique for Nondestructive

Evaluation of Composite Materials, A Review, Part 1

B. Kaiser: Schadensbewertung als Grundlage praxisnaher

Schwingfestigkeitsuntersuchungen, Teil 1. . . . . . . . . . . . . . . . 179

R. Best: Der Schadensparameter im Kerbgrundkonzept . . . . . . . . . . . 184

M. Klarskov, J. Jakobsen, A. Saarnak: Rheological Characterization of Waterborne Paints during the Film Formation . . . . . . . . . . . . . . 189

W. G. Fischer, P. Kusch: Curie-Punkt-Pyrolyse und Gaschromatographie . . 193

\section{Kurzberichte}

Zuverlässige Industrie-Prozeßrechner . . . . . . . . . . . . . . . . . 165

Atome werden sichtbar . . . . . . . . . . . . . . . . . . . . . . . . 169

Punktschweißverbindungen zerstörungsfrei prüfen . . . . . . . . . . . . . 173

Schwingfestigkeitsprüfungen mit Hochfrequenzpulsatoren . . . . . . . . 178

Digital geregelte Temperaturkammern . . . . . . . . . . . . . . . . . 178

Komplett ausgestattetes Wärmebildsystem. . . . . . . . . . . . . . . 178

Ergonomisch gestaltete Universalprüfmaschine . . . . . . . . . . . . . . 183

Variable Prüfmaschinen sind wirtschaftlicher . . . . . . . . . . . . . . . 188

Regel- und Auswertesystem für hydraulische Prüfmaschinen . . . . . . . 192

Hochauflösende Mikroskop-Photometersysteme . . . . . . . . . . . . . 195

$\begin{array}{ll}\text { Normen und Richtlinien } & 196\end{array}$

$\begin{array}{ll}\text { Bücher } & 197\end{array}$

$\begin{array}{ll}\text { Report } & 198\end{array}$

Impressum 200

Inserentenverzeichnis

\title{
Zur Titelseite:
}

Mit FRANK-Werkstoffprüfmaschinen sichern Sie die Qualität Ihrer Werkstoffe. Die Biegeprüfung an keramischen Werkstoffen und Glas-Isolierstoffen in Anlehnung an DIN/VDE 0335 Teil 2 wird mit der dazu entwickelten Probenaufnahme 01903, eingesetzt in einer Universalprüfmaschine durchgeführt. Sie liefert Aufschluß über E-Modul und Biegefestigkeit. Die präzise ermittelten Kraft- und Verformungswerte werden über Bildschirm-Rechner mit leistungsfähigen AnwenderProgrammen verarbeitet und dokumentiert.
KARL FRANK GMBH

Postfach 100362

D-6940 Weinheim

Telefon 06201/84-0

Teletex 6201991

Telefax 0620184290 
Biener

\section{...zum Fachgebiet}

\section{MPA Sachsen-Anhalt}

Zum 1. Januar 1991 wurde durch Erlaß des Ministers für Wirtschaft, Technologie und Verkehr des Landes Sachsen-Anhalt das dortige Landesmaterialprüfamt mit Sitz in Magdeburg gegründet. Die Bildung erfolgte durch Zusammenschluß der bereits viele Jahre tätigen unabhängigen Prüfstellen für Baustoffe und Metalle des ehemaligen Amts für Standardisierung, Meßwesen und Warenprüfung (ASMW) und dem Zentrallaboratorium des Straßenwesens Magdeburg. Das Landesmaterialprüfamt ist in die Abteilungen Baustoffe und Baukonstruktionen, Zentrallaboratorium für Straßenwesen, Werkstoff- und Bauteilprüfung (metallisch), Metall- und Umweltanalyse so-

$$
28_{1-}
$$

wie zerstörungsfreie Prüfung gegliedert und bauaufsichtlich als Prüf- und Überwachungsstelle vom Institut für Bautechnik, Berlin, anerkannt.

Bi (62961)

\section{DVM-Berichtsbände}

Die neue Übersichtsliste des vom DVM herausgegebenen Schrifttums enthält wieder zahlreiche Neuerscheinungen. Dabei handelt es sich um die Berichtsbände der 1990 vom Deutschen Verband für Materialforschung und -prüfung und seinen Arbeitskreisen veranstalteten Tagungen. Die Themen der entsprechenden Vortragsveranstaltungen waren:

- Moderne Betriebsfestigkeitsprüfung,

- Moderne Werkstoffe - Versagensmechanismen, Bruchmechanik, Betriebsfestigkeit,

\section{Arbeitsgruppen im DVM-Arbeitskreis „Bruchvorgänge"}

Im Arbeitskreis "Bruchvorgänge" des Deutschen Verbandes für Materialforschung und -prüfung e.V. (DVM) bestehen zur Zeit acht verschiedene Arbeitsgruppen, die sich mit unterschiedlichen Fragen bei Bruchvorgängen befassen. Ziel der Arbeit der Arbeitsgruppe Bruchmechanik an Schweißverbindungen ist die Diskussion aktueller Forschungsarbeiten zu Themen der Schweißmechanik unter Berücksichtigung werkstoffspezifischer, mechanischer sowie verfahrens- und prüftechnischer Gesichtspunkte. Außerdem werden in der Arbeitsgruppe unter Leitung von Dr. J. G. Blanck die Aktivitäten des DVS und anderer Verbände im Bereich Bruchmechanik an Schweißverbindungen koordiniert. Die von Prof. Dr. W. Dahl betreute Arbeitsgruppe Mikromechanismen will einen Beitrag zur qualitativen und quantitativen Bewertung des Einflusses der Mikrostruktur auf Bruchvorgänge leisten. Dazu werden auf europäischer Ebene verschiedene Ringversuche durchgeführt.

Primäres Ziel der neu eingerichteten Arbeitsgruppe Ermüdungsrißausbreitung_Kennwertermittlung unter Leitung von Dr. H. Döker ist die Erweiterung des Kenntnisstandes auf dem $\mathrm{Ge}$ biet der Ermüdungsrißausbreitung und der Ermittlung entsprechender Kennwerte. Die Erarbeitung von Verbesserungsvorschlägen für die bruchmechanische Charakterisierung von Wärmeeinflußzone und Schweißgut und eine Überarbeitung des DVM-Merkblattes 002 "Ermittlung von Rißinitiierungswerten und Rißwiderstandskurven bei
Anwendung des J-Integrals und der Rißöffnung CTOD" stehen bei der Arbeitsgruppe Kennwertermittlung und Anwendung der Bruchmechanik im Vordergrund. Die von Dr. R. Kienzler geleitete Arbeitsgruppe Integralkriterien - Bruchkriterien befaßt sich mit der experimentellen und theoretischen Absicherung des derzeitigen Standes in der Ermittlung von Werkstoffkenngrößen der elastisch-plastischen Bruchmechanik.

In der Arbeitsgruppe Instrumentierter W. Klemm) soll die Anbindung dieses Versuchs an die Bruchmechanik vorangetrieben sowie neue Meß- und Auswerteverfahren für den Kerbschlagbiegeversuch entwickelt und erprobt werden. Die siebte Arbeitsgruppe Mixed-Mode-Probleme bearbeitet Fragen der komplexen Rißbeanspruchung. Unter der Leitung von Prof. $\mathrm{H}$. A. Richard werden u. a. die Themenkreise mehrachsige Bauteilbelastung, Kriterien zur Burchvorhersage, Ermüdungsrißwachstum bei komplexer Beanspruchung sowie numerische Ermittlung von Beanspruchungsgrößen behandelt. Eine neue regionale Magdeburger Arbeitsgruppe Bruchmechanik wird sich unter der Leitung von Dr.Ing. G. Dehne jährlich treffen.

Alle Arbeitsgruppen führen in regelmäßigen Abständen Sitzungen durch, bei denen neue Erkenntnisse diskutiert und Erfahrungen ausgetauscht werden können. Alle am jeweiligen Thema Interessierten sind zur Mitarbeit aufgefordert. Kerbschlagbiegeversuch (Leitung: Dr.
Mikrostrukturelle und mikroanalytische Charakterisierung in Werkstoffentwicklung und Qualitätssicherung,

Aussagefähigkeit von Prüfergebnissen für das Verarbeitungs- und Bauteilverhalten,

- Prüfen neuer Werkstoffe, Prüfmethoden - Anwendung und Aussagefähigkeit.

Außerdem ist im vergangenen Jahr das DVM-Merkblatt 003 „Einfluß verschiedener Oberflächenbehandlungsverfahren auf die wichtigsten Bauteileigenschaften" neu erschienen. Die aktuelle Übersichtsliste, die 73 Schrifttumsangaben enthält, kann beim DVM, Berlin, bezogen werden.

Bi (62958)

\section{...aus den Unternehmen}

\section{CMMs from Lk Ltd.}

LK Tool Company Limited, Castle Donington, England, was founded in 1961 as a sub-contractor in the precision machining sector of the engineering industry. In 1969, the Company manufactured its own computerised co-ordinate measuring machine (CMM) and is now a world leader in the design, manufacture and marketing of CMM's. CMM's are produced by LK in two basic models vertical spindle (bridge type) and horizontal spindle types. Machines are available in manual, computer assisted control, or under full CNC computer control

Innovative developments in machines for special applications have resulted from the Company's willingness to accept challenges posed by its customers - particularly in the aerospace industry. The Company has manufactured some of the largest machines in the world for large aerospace and defence applications. Lk Ltd. has been particularly involved in the design and manufacture of very large, precision machines for the ultra-sonic, non-destructive testing of composite laminate materials used in the construction of advanced aircraft.

Currently LK Ltd. has approximately 200 employees (120 in UK) and have worldwide sales averaging $£ 22-£ 24$ million annually.

Bi (62913)

\section{Carl-Zeiss-Stiftung entsteht}

Am 7. November 1990 haben sich die Unternehmen Jenaer Glaswerk GmbH, Jenaoptik Carl Zeiss Jena GmbH, Schott Glaswerke, Mainz, und Carl Zeiss, Oberkochen, auf eine Rahmenvereinbarung über Maßnahmen zur Zusammenführung der Jenaer Gesellschaften mit den Stiftungsunternehmen in Mainz und 
Oberkochen und die Zusammenarbeit bei der Sanierung der Jenaer Firmen geeinigt. Die Vereinbarung wurde am 19. und 20. November 1990 von den Aufsichtsgremien der Unternehmen Schott Glaswerke, Mainz, und Carl Zeiss, Oberkochen, verabschiedet. Sie sieht u. a. vor, daß die Unternehmen bei der Bewältigung der wirtschaftlichen Probleme der Jenaer Betriebe partnerschaftlich zusammenarbeiten und im
Rahmen eines Kooperationsvertrages schrittweise wieder in eine Stiftung zusammenwachsen. Wichtiger Bestandteil des Sanierungskonzeptes soll die Sicherung möglichst vieler Arbeitsplätze sein. Die abgeschlossene Rahmenvereinbarung ist ein großer Schritt auf dem Weg in eine gemeinsame Zukunft der Unternehmen, die sich in einer Carl-Zeiss-Stiftung zusammenschließen wollen.

\section{Dynisco erweitert Vertriebs- programm}

Dynisco, Heilbronn, Hersteller von Massedrucksensoren und Folgeelektronik für die kunststoffverarbeitende Industrie hat sein bereits umfangreiches Vertriebsprogramm erweitert. Ab sofort führen alle Dynisco Niederlassungen Schmelzeindex Prüfgeräte der Fa. Kayeness im Programm. Mit diesen Geräten können Materialanlieferungen auf

\section{Aerospace-Workshop bei Dr. Förster}

Speziell Wartungsfachleute aus dem Bereich der zivilen und militärischen Luftfahrt waren im Februar zu jeweils eintägigen Workshops beim Institut Dr. Förster, Reutlingen, zu Gast. Im Zentrum stand die Wirbelstrom-Prüfung zum Aufspüren von Rissen in und unter der Oberfläche, in Bohrungen und auch als Korrosionsschaden. Eine gute Gelegenheit für die rund 20 Teilnehmer, ihr Basiswissen über Wirbelstrom-Prüfung und verwandte, zerstörungsfreie Prüfverfahren aufzufrischen und vor allem um neue Prüftechniken und Geräte kennenzulernen bis hin zu rechnergestützten Prüfeinrichtungen mit komfortabler Bildverarbeitung. Die neuzeitlichen Metho- den der Rechnerunterstützung werden zu einer sehr weitgehenden Form der Meßdatenaufbereitung genutzt, wie sie bisher nicht möglich schien. Bedienbarkeit und Programmierung der Prüfgeräte sind wesentlich vielseitiger und dabei einfacher geworden, die Interpretation der Meßergebnisse wird objektiver

Erstmals hatte Förster diesen Workshop für die Luftfahrtindustrie 1990 erfolgreich in Singapur durchgeführt, gleiche Veranstaltungen folgten in England im Mai und Frankreich im März. Angesichts des drastisch zunehmenden Alters von Flugzeugen (Bild 1) und der drastisch gestiegenen Prüfaufwendungen (Bild 2: für eine Flugstunde Jumbo müssen 16
Mann-Stunden Wartung aufgebracht werden) mißt Förster diesem Gebiet große Bedeutung zu. Insgesamt verzeichnet das Unternehmen derzeit rund $70 \mathrm{Mio}$ Umsatz aus Deutschland, 90 Mio. weltweit. $70 \%$ der Produktion gelangen in den Export mit Schwerpunkten Westeuropa, Japan und Amerika. Man beschäftigt derzeit 365 Mitarbeiter in Deutschland und 530 weltweit unter Einschluß zahlreicher Tochtergesellschaften. Dem zunehmenden Mangel an Fachkräften und der steigenden Komplexität von Prüfaufgaben und Gerätemöglichkeiten hat man jüngst Rechnung getragen durch den Bau eines Schulungszentrums für Prüfpraktiker in kleinen Gruppen. (62942)

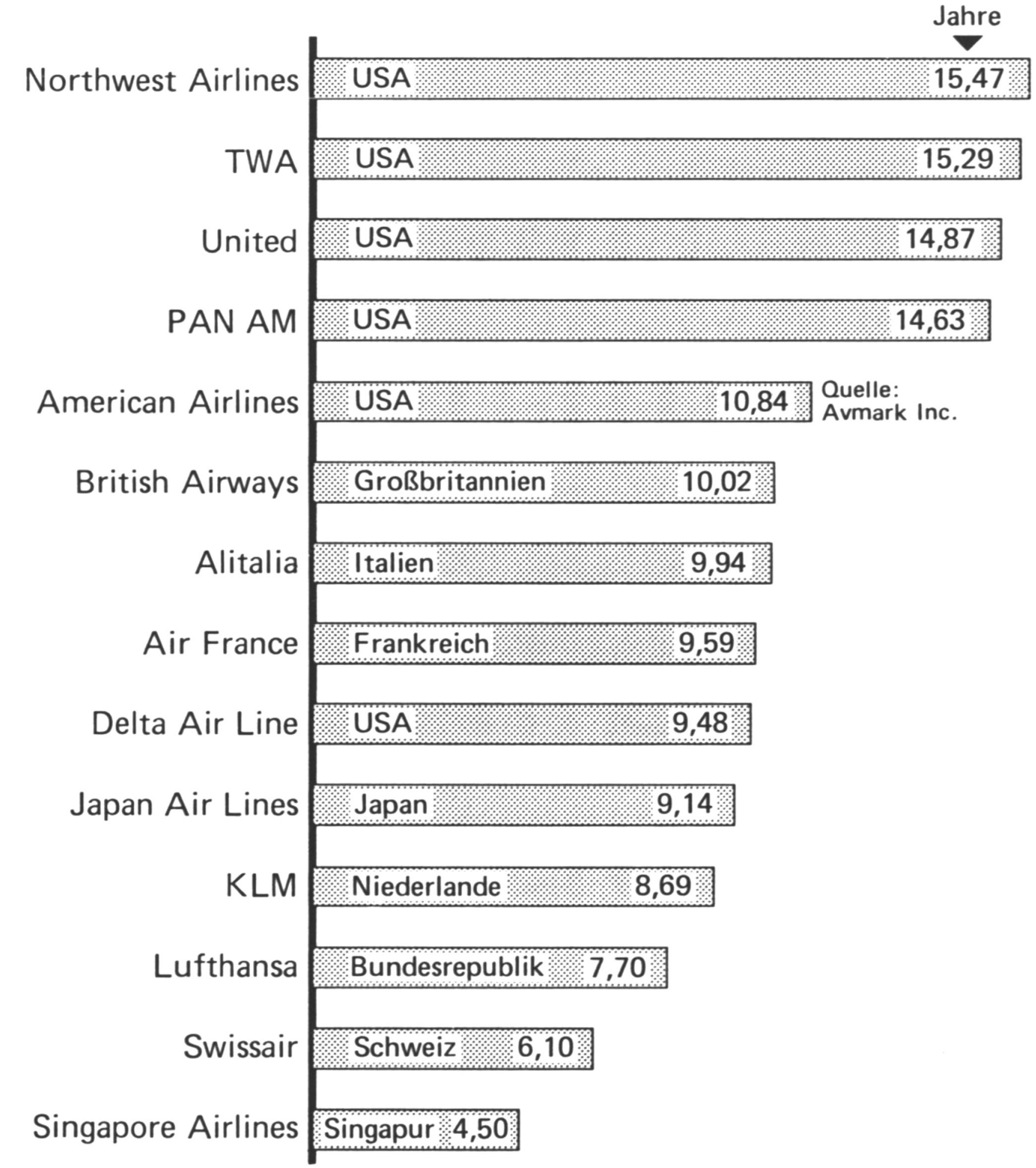

Bild 1. Das zivile Fluggerät wird immer älter: Durchschnittsalter bei verschiedenen Gesellschaften am 1.7.1988 (Quelle: Der Spiegel)

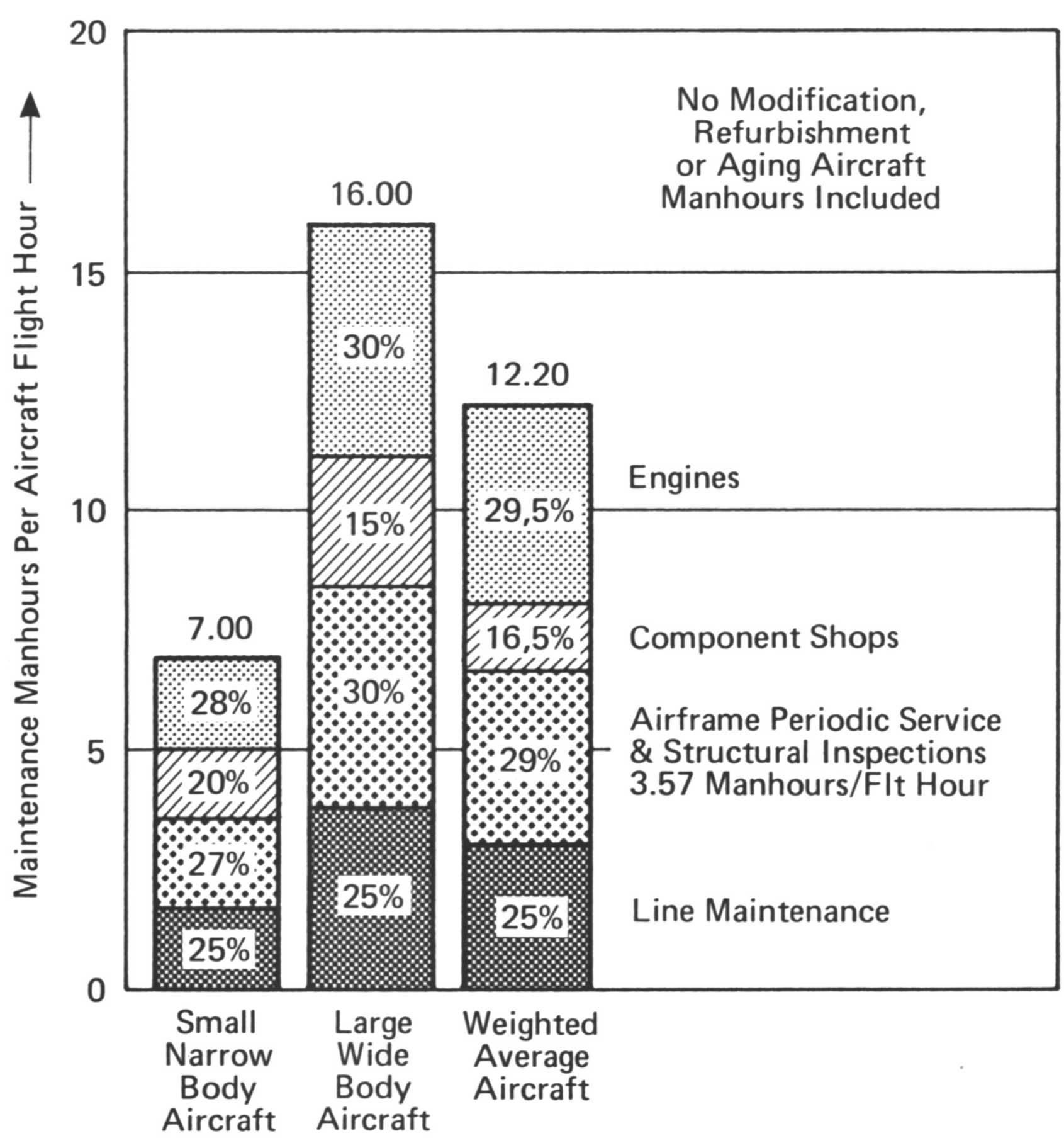

Bild 2. Hoher Wartungsaufwand, insbesondere bei Großflugzeugen (US Airline Maintenance, nach Airclaims) 


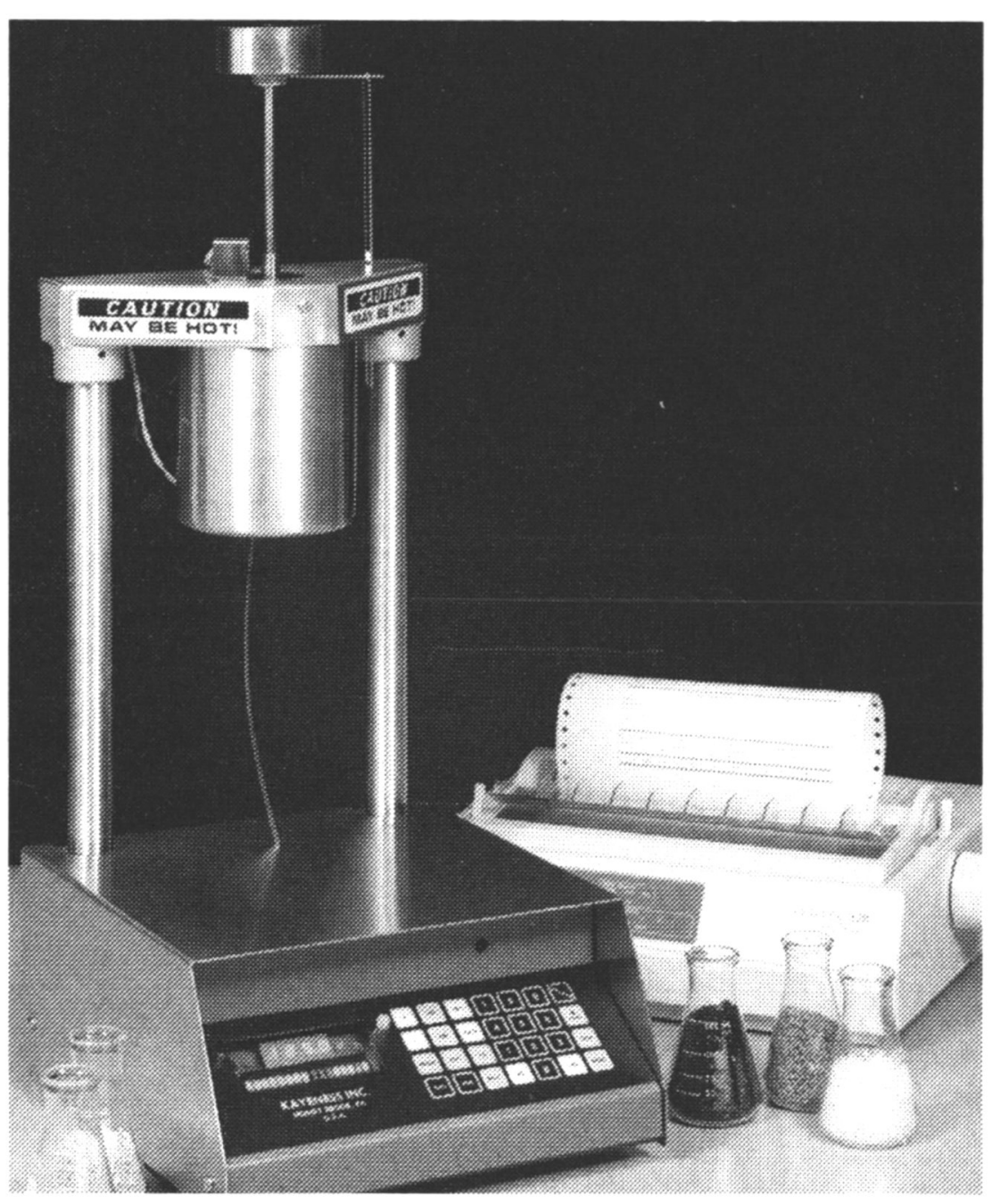

die Einhaltung der Spezifikationen und auf Konsistenz der Lose überprüft werden. Der eingebaute Microcomputer erlaubt einen automatischen Testablauf und eine einfache Kontrolle der Prüfbedingungen bei geringem Aufwand durch das Bedienungspersonal.

Bi (62947)

\section{Spectro gründet Gemeinschafts-}

\section{unternehmen}

Mit Spezialisten aus Dresden und Berlin gründete der deutsche Spektrometerhersteller Spectro Analytical Instruments $\mathrm{GmbH}$, Kleve, die Firma Spectro X-Ray Instruments $\mathrm{GmbH}$ mit Sitz in Berlin. Produziert wird ein neuartiges energiedispersives Röntgenfluoreyszenzspektrometer. Geschäftsführer des neugegründeten Unternehmens ist Dr. Michael Haschke, der vorher einen Zweigbetrieb im „Zentrum für wissenschaftlichen Gerätebau" an der Akademie der Wissenschaften der ehemaligen DDR leitete.

Bi (62948)

\section{DGZfP in Zahlen}

Die von der Deutschen Gesellschaft für Zerstörungsfreie Prüfung für das Jahr 1990 veröffentlichten Zahlen über Mitglieder und Aktivitäten lassen gegenüber dem Vorjahr ein Wachstum in allen Bereichen erkennen. Die Zahl der Mitglieder lag am Ende des vergangenen Jahres

\section{ZfP-Symposium bei Nukem}

Am 27. und 28.9.1990 fand bei der Nukem GmbH, Alzenau, das 7. Internationale Symposium "Zerstörungsfreie Werkstoffprüfung" statt, an dem etwa 150 Gäste teilnahmen und das in einem zweijährigen Turnus veranstaltet wird. Am ersten Tag des Symposiums referierten traditionsgemäß Fachleute über neue Methoden und Anwendungen der zerstörungsfreien Werkstoffprüfung. Der zweite Tag stand im Zeichen neuer Nukem-Entwicklungen auf diesem Gebiet. Dazu gehört eine speziell für die Fehler- und Dimensionsprüfung warmgewalzter Stahlrohre entwickelte Ultra-
schall-Rotationsprüfanlage, die zur Prüfung hochbelasteter Rohre eingesetzt wird. Weiter wurden Ultraschallanlagen zur Prüfung großflächiger Flugzeugbauteile aus Kohlefaser-Verbundwerkstoffen und für die Wanddicken-Messung von warmgewalzten Stahlrohren vorgestellt.

Zu den Neuheiten gehört auch eine Anlage zur Prüfung von Rohrenden, in der gleichzeitig Fehler festgestellt werden können und die Messung der Wanddicke möglich ist. Dies ist durch die gleichzeitige Nutzung eines gemeinsamen $\mathrm{Ma}$ gnetfeldes durch zwei unterschiedliche Prüfkopftypen möglich.

Bi (62906)

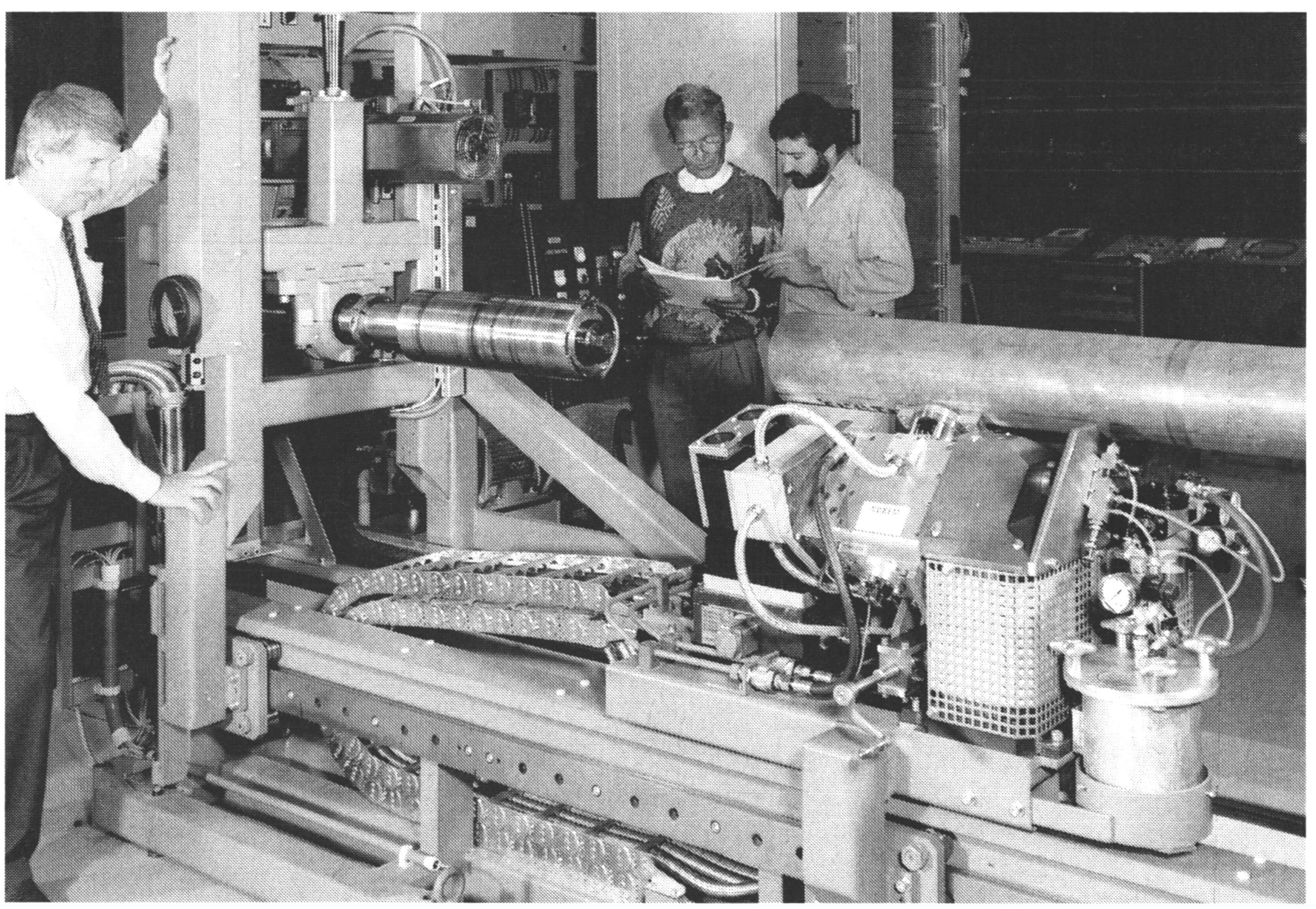

mit 1136 um über 8\% höher als im vorhergehenden Jahr. Während die 30 Vorträge bei der Jahrestagung von 580 Teilnehmern gehört wurden, waren bei den 115 Arbeitskreistagungen im Jahr 1990 3256 Interessenten anwesend.

Auch im Bereich der Aus- und Weiterbildung war die DGZfP wieder sehr aktiv. Die 91 angebotenen Kurse und Praktika wurden von 1559 Teilnehmern genutzt. Diese stuften bei einer Befragung die Vorträge und Übungen als gut bis befriedigend ein. Auch Organisation und Ausgewogenheit der Veranstaltungen, die Qualität der Unterlagen und der äußere Rahmen wurden als gut bewertet. Damit wird den Veranstaltern ein hervorragendes Zeugnis für ihre Arbeit ausgestellt.

Bi (62938)

\section{Herfurth übernimmt Graetz}

Die Herfurth $\mathrm{GmbH}$, Hamburg, hat zum 1.1.1991 den Geschäftsbetrieb der bisherigen Graetz Strahlungsmeßtechnik $\mathrm{GmbH}$, Altena, durch die in Gründung befindliche Strahlungsmeßtechnik GmbH Altena, einer 100\% igen HerfurthTochter, übernommen. Die Übernahme ist aufgrund der langjährigen engen und erfolgreichen Zusammenarbeit zwischen Graetz und Herfurth zustandegekommen. Sie gewährleistet eine Fortsetzung und Weiterentwicklung des Graetz Programms Strahlungsmeßtechnik ebenso wie die Betreuung des bisherigen Kundenstamms. Die bekannten Dosisleistungsmeßgeräte und Personendosimeter werden auch künftig unter dem Namen "Graetz" verkauft, wobei der Gesamtvertrieb zentral durch die Herfurth $\mathrm{GmbH}$ erfolgt.

Bi (62949)

\section{Schenck AG erhöht Umsatz}

Der Konzernumsatz der Carl Schenck AG, Darmstadt, ist im Geschäftsjahr 1989/90 (30.9.) um 3\% auf 1,04 Mrd DM gestiegen. Wie das Maschinenbauund Elektronikunternehmen mitteilte, entfielen davon 707 Mio. DM (+ 11\%) auf die Carl Schenck AG. Als Folge dieser guten Entwicklung ist die Zahlung einer erhöhten Dividende von 7,50 DM bei Stammaktien und 8,50 DM bei Vorzugsaktien vorgesehen.

Bi (62960)

\section{...zur Person}

Prof. Dr.-Ing. Hans Diergarten, langjähriger Kurator der Bundesanstalt für Materialforschung und -prüfung (BAM) und Ehrenmitglied des DVM-Vorstandes, ist am 9. Februar $1991 \mathrm{im}$ Alter von 89 Jahren verstorben. Der 1901 geborene Hans 
Diergarten studierte ab 1922 in Aachen Eisenhüttenwesen. Nach Abschluß seines Studiums und erfolgter Promotion trat er 1929 in die Vereinigten Kugellagerfabriken $A G$ in Schweinfurt ein und wurde dort bereits 1942 zum Direktor ernannt. 1956 übertrug ihm die Würzburger Universität die Honorarprofessur für Werkstofftechnik-Metallkunde, die er am Institut für physikalische Chemie wahrnahm. Für seine umfangreichen Arbeiten auf den Gebieten der qualitativen und quantitativen Metallographie bei Stählen sowie der Tribologie hat Prof. Diergarten zahlreiche Ehrungen erfahren. Dazu gehören u. a. die Verleihung der Ehrendoktorwürde der TU Berlin, der Erich-Siebel-Gedenkmünze und des Bundesverdienstkreuzes. Bi (62959)

\section{Prof. Dr. rer. nat. Dr.-Ing. e.h. Hubert Gräfen}

Am 26. Januar 1991 vollendete Hubert Gräfen sein 65. Lebensjahr. Er stellte mit einer Diplomarbeit über die Spannungsrißkorrosion unlegierter Stähle bereits frühzeitig die Weichen für seine Zukunft. Es erscheint daher folgerichtig, daß er im Rahmen seiner ersten Anstellung eine Abteilung für Korrosion in der Materialprüfung der BASF AG aufbaute. In diese Zeit hinein fiel auch seine Dissertation über das Ausscheidungsverhalten von Nickellegierungen, mit der er den Grundstein für eine Gruppe von hochbeständigen Werkstoffen der Chemietechnik legte. 1970 übernahm er die Leitung der Materialprüfung der Bayer AG, 1976 wurde er zum Abteilungsdirektor ernannt.

Seine Hoschschullaufbahn verlief ebenso geradlinig: 1969 erhielt er seinen ersten Lehrauftrag, 1972 habilitierte er sich und 1977 schließlich wurde er vom Kultusminister des Landes Niedersachsen zum außerplanmäßigen Professor ernannt.

Hubert Gräfens Verdienste erstrecken sich nicht nur auf das Erarbeiten wichtiger wissenschaftlicher Erkenntnisse und ihre Umsetzung in die Praxis, sondern auch auf ihre Verbreitung. So ist er der Autor von weit mehr als 200 Publikationen und hat mit seiner Tätigkeit in zahlreichen technischen Organisationen seine Erfahrung uneigennützig in den Dienst der Normung und Ausbildung gestellt. Stellvertretend genannt seien sein Vorsitz im Vorstand des DVM 1987 1990, sein Vorsitz in der Fachgesellschaft Werkstofftechnik des VDI und seine Aktivität im Lenkungsausschuß des vom BMFT finanzierten und von der Dechema verwalteten Forschungsprojektes Korrosion und Korrosionsschutz.

Am 23. Oktober 1989 wurde Hubert Gräfen für seine Verdienste auf den Ge- bieten Korrosionswissenschaft und Metallkunde vom Dekan der Fakultät für Bergbau, Hüttenwesen und Maschinenwesen an der Technischen Universität Clausthal mit der hohen akademischen Auszeichnung des Dr.-Ing. ehrenhalber geehrt.

Der Ehrendoktorwürde gingen mehrfache Auszeichnungen voraus. Die DINEhrennadel und die Beuth-Gedenkmünze des DNA, die Wellinger-Medaille des VDI und die Achema-Plakette in Titan der Dechema.

Ende 1988 trat Hubert Gräfen in den verdienten Ruhestand. Allerdings ließ inm die Fachwelt bislang keine Gelegenheit, diesen Begriff allzu wörtlich auszulegen. Für seinen weiteren Lebensweg begleiten ihn die besten Wünsche seiner Freunde und Kollegen.

Ubbo Gramberg (62941)

Dr.-Ing. Hans Siegwart, langjähriges Vorstands-Mitglied im DVM, ist am 28.2.1991 im Alter von 79 Jahren verstorben. Hans Siegwart studierte Maschinenbau an der TH Darmstadt und war anschließend dort als Mitarbeiter am Institut für Werkstoffkunde und der Staatlichen Materialprüfungsanstalt tätig. Er befaßte sich dort mit dem Verhalten von Kunststoffen unter betrieblichen Beanspruchungsbedingungen und promovierte über ein Thema aus diesem Bereich. Nach dem Krieg war Dr. Siegwart maßgeblich am Wiederaufbau der Darmstädter Materialprüfungsanstalt beteiligt, bevor er 1954 zur Daimler-Benz AG nach Stuttgart wechselte, wo er Leiter der Zentralen Werkstofftechnik wurde. Durch seine Mitarbeit in zahlreichen Verbänden, Ausschüssen und Gremien war sein Name in der gesamten Fachwelt bekannt.

Bi (62955)

\section{Tagungen, Seminare}

\section{Metallographie-Tagung 1991}

11. - 13.9.1991, Friedrichshafen

Die Themen dieser Tagung umfassen Präparation, Gefügedarstellung und -interpretation bei metallischen Werkstoffen, nichtmetallischen Werkstoffen und Verbundwerkstoffen im Rahmen von Materialforschung und -entwicklung, Qualitätssicherung und Schadensanalyse. Als Schwerpunktthemen sind vorgesehen Luftfahrtwerkstoffe, Hochtemperaturwerkstoffe sowie Verschleißund Korrosionsschutzschichten. Weitere Informationen bei Deutsche Gesellschaft für Materialkunde e.V., Adenaueralle 21, D-6370 Oberursel.

\section{Weiterbildung}

\section{interflux}

Kurs Magnetpulver-Prüfung, 11. - 13.9. 1991, Essingen. Eine anschließende Qualifikation zum Prüfwerker nach DGZfPRichtlinien ist möglich. Weitere Informationen bei interflux Prüfgeräte-Vertrieb $\mathrm{GmbH}$, Postfach 17, D-7087 Essingen.

\section{Deutsche Gesellschaft für Material- kunde e.V.}

Qualitätssicherung in der Werkstoffprüfung, 18. - 20.9.1991, Siegen. Dieses Fortbildungspraktikum zeigt, wie die einfachen Prüfverfahren zur Ermittlung der Werkstoffeigenschaften durch Härteprüfung, durch den Zugversuch und den Kerbschlagversuch mittels Rechnereinsatz bei Versuchssteuerung und Auswertung der Prüfdaten weiterentwickelt werden. Dieses Aufgabengebiet wird in Seminarvorträgen mit Erfahrungs- und in Laborübungen mit Demonstrationen von Prüfverfahren vermittelt. Weitere Informationen bei Deutsche Gesellschaft für Materialkunde e.V., Adenauerallee 21, D-6370 Oberursel.

\section{Krautkrämer}

Kursusprogramm: Werkstoffprüfung mit Ultraschall.

Einführung in die Grundlagen:

$\begin{array}{ll}\text { 26. }-30.8 .1991 & 25 .-29.11 .1991\end{array}$

23. $-27.9 .1991 \quad 9 .-13.12 .1991$

21. -25.10 .1991$

Prüfung: Prüfwerker ZfP - Bereich Ultraschall:

$\begin{array}{rr}\text { 5. } 9.1991 & 28.10 .1991 \\ \text { 30. } 9.1991 & 16.12 .1991\end{array}$

Bewertung von Ungänzen:

9. -13.9 .1991$

7. -11.10 .1991$

USD 10-Praktikum:

17. - 19. 9.1991

29. -31.10 .1991$

2. -4.12 .1991$

Ultraschall-Wanddickenmessung

10. - 11. 6.1991

4. - 5.11.1991

Level-Kurse und Prüfungen in englischer Sprache.

Level 1 mit Schweißnahtprüfung

24.6.-1.7.1991.

Prüfung: Level 1 nach SNT-TC-1A:

2.7.1991.

Level 2 mit Schweißnahtprüfung

3. - 10.7.1991.

Prüfung: Level 2 nach SNT-TC-1A:

11.7.1991

Weitere Informationen bei Krautkrämer GmbH \& Co., Kursusabteilung, RobertBosch-Str. 3, 5030 Hürth 5.

(62964) 